Article

\title{
Development of the Dyskinesia Impairment Mobility Scale to Measure Presence and Severity of Dystonia and Choreoathetosis during Powered Mobility in Dyskinetic Cerebral Palsy
}

\author{
Saranda Bekteshi ${ }^{1, *}\left(\mathbb{D}\right.$, Marco Konings ${ }^{1}$, Ioana Gabriela Nica ${ }^{2}$, Sotirios Gakopoulos ${ }^{3}$, \\ Inti Vanmechelen ${ }^{1}$, Jean-Marie Aerts ${ }^{2}$, Hans Hallez ${ }^{3}$ and Elegast Monbaliu ${ }^{1}$ (D) \\ 1 KU Leuven, Bruges Campus, Department of Rehabilitation Sciences, Research Group for \\ Neurorehabilitation, 8200 Bruges, Belgium \\ 2 KU Leuven, Department of Biosystems, Division of Animal and Human Health Engineering, Measure, \\ Model and Manage Bioresponse (M3-BIORES), 3001 Leuven, Belgium \\ 3 KU Leuven, Bruges Campus, Department of Computer Science, Mechatronics Research Group, \\ 8200 Bruges, Belgium \\ * Correspondence: saranda.bekteshi@kuleuven.be; Tel.: +32-5066-4992
}

Received: 20 July 2019; Accepted: 20 August 2019; Published: 23 August 2019

check for updates

Featured Application: The Dyskinesia Impairment Mobility Scale (DIMS) is a reliable tool to measure presence and severity of dystonia and choreoathetosis during powered mobility in dyskinetic cerebral palsy. In clinical practice, the DIMS can assist a better structure of the mobility training programs to shorten learning curves and a better evaluation of mobility training progress. In research, the DIMS can be used to explore clinical patterns during powered mobility and evaluate future mobility intervention studies.

\begin{abstract}
The majority of individuals with dyskinetic cerebral palsy cannot use powered mobility with a joystick, due to the lack of manual abilities by the severe presence of dystonia and choreoathetosis. Reliable measurements of these movement disorders is indispensable for good evaluation towards evidence-based insights during powered mobility. This study aimed to develop and assess the Dyskinesia Impairment Mobility Scale (DIMS), a video-based tool to measure presence and severity of dystonia and choreoathetosis during powered mobility. DIMS was measured for the neck and arms region during five mobility tasks. Interrater reliability, test-retest reliability, internal consistency and concurrent validity of the DIMS were assessed. Interrater reliability coefficients ranged between 0.68 and 0.87 for the total DIMS, and the dystonia and choreoathetosis subscales. Test-retest reliability was moderate to excellent (range 0.51-0.93) while Cronbach's alpha was good (range 0.69-0.81) for the total scale and subscale scores. Concurrent validity showed during mobility tasks significant correlations with rest postures in the arm region, and with requested but voluntary activity in the neck region. The DIMS reliably measures the presence and severity of the movement disorders during powered mobility, increasing insights into the underlying mechanisms of independent mobility. This scale may therefore be a promising tool to evaluate mobility training.
\end{abstract}

Keywords: powered mobility; dyskinetic cerebral palsy; dystonia; choreoathetosis; mobility scale; movement disorder; children; youth; reliability; validity

\section{Introduction}

Impaired mobility is the leading cause of reduced functionality, restricted participation levels and decreased activity levels in children and youth with severe movement disabilities, resulting in, among 
others, social isolation, anxiety and depression [1-3]. Introducing powered mobility wheelchairs to children with severe motor limitations has shown to improve their psychosocial and cognitive skills while increasing independence, self-exploration and intuition without any negative impact on their motor development [4]. Therefore, mobility training is of major importance starting at a young age [5].

In the child-population, cerebral palsy is the most common neuromotor disability, with a prevalence of 1.7 per 1000 livebirths [6] and is categorized into three groups: Spastic, dyskinetic and ataxic [7]. Dyskinetic cerebral palsy (DCP) is the second largest and most limiting CP group [8]. DCP is characterized by two complex movement disorders: Dystonia (i.e., involuntary movements, distorted voluntary movements and abnormal postures due to sustained muscle contractions) and choreoathetosis (i.e., chorea defined as rapid, involuntary, often fragmented movements and athetosis defined as slower and constantly changing movements) [8]. In DCP, dystonia and choreoathetosis are also present as overflow movements, defined as unintentional contractions of muscles different from the ones used during a goal-directed movement [9]. More than 70\% of the DCP population presents with the highest levels of severity in gross motor functioning and fine manual abilities [10]. As a result, children with DCP are unable to use powered mobility wheelchairs with a conventional joystick, hence leaving them heavily dependent on caregivers' assistance $[4,8,11]$.

Alternate steering methods such as switches or head arrays for powered mobility are proposed in literature as a way to achieve independence for children with severe gross and manual limitations [12]. In this respect, in DCP, the basal movements of the head and the feet are better controlled than the movements of the arms [8]. Therefore, clinical practice widely supports the use of a head/foot steering system as the best option to promote independent powered mobility for children with $\mathrm{DCP}$, where the head movements are used to steer the wheelchair to the right and left and the feet are used to drive the wheelchair forward and backward. In general, there is little evidence supporting the best methods to train children to use a powered wheelchair and a lack of comprehensive training results in longer, time-consuming and unstructured motor learning and skill acquisition processes $[4,13]$.

Thus, evidence-based knowledge into the different stages of powered mobility, from learning towards self-exploration remains scarce in DCP and mobility training so far is based only on clinical expertise. To increase insights into the potential use and learning process of powered mobility wheelchairs for children with $\mathrm{DCP}$, the process of learning to use it, operation of the system, the impact of movement disorders and environmental factors need to be further explored. These insights would contribute to a better understanding of the mobility limitations in this population, and assist in tailoring of better powered mobility training programs to shorten learning curves.

Whereas the characterizing dystonia and choreoathetosis movement disorders seem to be the biggest limiting concepts of powered mobility in DCP [8], it is important to be able to assess the presence and severity of these movement disorders during powered mobility tasks. That is, it is stipulated that an increase in severity causes higher distortion of voluntary movements and higher presence of involuntary/overflow movements, which might result in longer and more difficult powered mobility skills training. Thereby, increased insights in the severity of dystonia and choreoathetosis during powered mobility may generate knowledge to develop more straightforward mobility training guidelines and assist powered mobility training by shortening learning curves.

To date, presence and severity of dystonia and choreoathetosis are measured using the Dyskinesia Impairment Scale (DIS) [9,14-16], a video-based tool which measures the movement disorders during requested but voluntary activities and rest postures. The DIS measures requested but voluntary activities in a controlled environment and may, therefore, not provide information about the occurrence of the movement disorders in a more real-life context, such as steering a powered wheelchair using a head/foot steering system. This implies that there is a necessity for a reliable and valid assessment tool that will generate insights on the presence and severity of movement disorders during daily-life activities. The DIS has shown high reliability and validity in measuring dystonia and choreoathetosis in DCP $[14,15]$. Thereby, an adapted protocol of the DIS which will reliably measure the movement 
disorders during powered mobility could be the solution to fill in the gap in the current existing assessment tools.

Therefore, this study aimed to (1) develop an adapted standardized protocol of the DIS which will measure presence and severity of dystonia and choreoathetosis during powered mobility tasks in individuals with DCP, and (2) to assess the reliability and validity of this protocol. Serving that purpose, the new scale will be named the Dyskinesia Impairment Mobility Scale (DIMS).

\section{Materials and Methods}

\subsection{Development of the Dyskinesia Impairment Mobility Scale (DIMS)}

In the first step towards developing the adapted DIMS protocol, the DIS was examined thoroughly for dystonia and choreoathetosis characteristics which would be relevant to be included in the DIMS [9,14-16]. The DIS measures the presence and severity of dystonia and choreoathetosis over 12 body regions; i.e., central body (eyes, mouth, neck and trunk) and limbs (upper and lower, proximal and distal limbs) [14]. Dystonia and choreoathetosis are assessed at rest and during two requested voluntary activities, both for duration (i.e., the amount of time that dyskinesia is present) and amplitude (i.e., the maximum range of motion (ROM) achieved due to dyskinesia) [14]. Duration and amplitude are scored on a five-point ordinal scale ranging from 0 to 4 . Summation of region scores gives a total rest score, ranging 0-96, and a total action score, ranging 0-192. A total dystonia subscale score and total choreoathetosis score is obtained by the sum of the total rest and action score with a range from 0-288. A diagram of the DIS protocol is shown in Appendix A, Figure A1.

For the DIMS, a similar protocol as for the DIS was adopted (Figure 1). The final DIMS protocol is a video-based protocol which consists of the dystonia subscale (DIMS-D) and choreoathetosis subscale (DIMS-CA). Both subscales measure duration (i.e., the amount of time that dystonia and choreoathetosis were present during the powered mobility task) and amplitude (i.e., the maximum ROM achieved due to dystonia and choreoathetosis) during five different powered mobility tasks. Scoring of duration and amplitude ranges from 0 to 4 (Table 1). For the duration factor, score 0 means the movement disorder is absent during powered mobility, score 1 means occasionally present (i.e., less than $10 \%$ ), score 2 means frequently present (i.e., between 10 and $50 \%$ of the time), score 3 means mostly present (i.e., between 50 and $90 \%$ of the time) and score 4 means always present (more than $90 \%$ of the time). For the amplitude factor, the range of percentages is the same as for the duration factor with score 0 assigned when the movement disorder is absent during powered mobility, score 1 when the movement disorder is present in a small ROM, score 2 when present in moderate ROM, score 3 when present in submaximal ROM and score 4 when present in maximal ROM. The DIMS is comprised of the neck region, representing the voluntary movements used to steer the wheelchair to the right and to the left, and the arm region, representing the dystonia and choreoathetosis overflow movements during mobility tasks. For the arm region, a distinction is made between left and right and proximal and distal parts. As such, the DIMS is comprised of five body regions: neck, right arm proximal (i.e., arm RP), left arm proximal (i.e., arm LP), right arm distal (i.e., arm RD), and left arm distal (i.e., arm LD). Even though the feet are used to accelerate and brake with the wheelchair, they are strapped during powered mobility manifesting minimal movement, and were therefore excluded from the final DIMS protocol. 


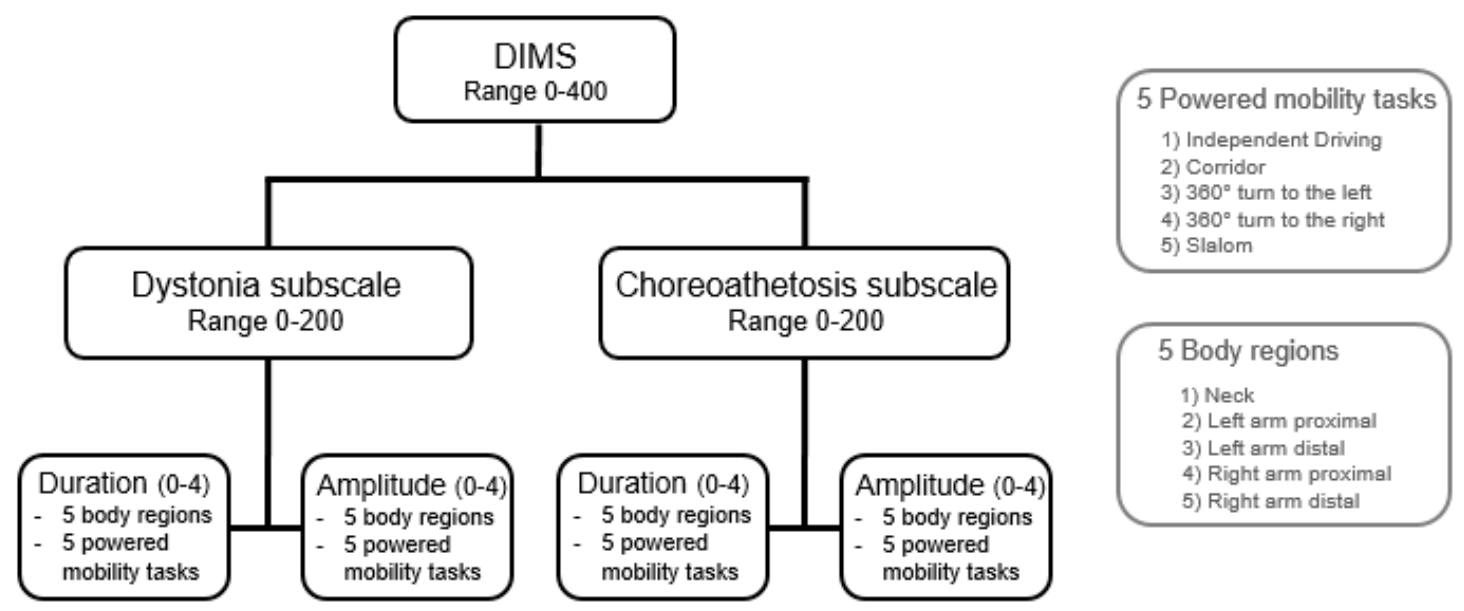

Figure 1. Diagram of the Dyskinesia Impairment Mobility Scale (DIMS) protocol.

Table 1. Scoring protocol of the Dyskinesia Impairment Mobility Scale (DIMS) duration and amplitude during powered mobility.

\begin{tabular}{ccc}
\hline & Duration & Amplitude \\
\hline Score $\mathbf{0}$ & D/CA is absent & D/CA is absent \\
Score $\mathbf{1}(<\mathbf{1 0} \%)$ & D/CA is occasionally present & D/CA is present in small ROM \\
Score $\mathbf{2}(\mathbf{1 0}<\mathbf{5 0} \%)$ & D/CA is frequently present & D/CA is present in moderate ROM \\
Score $\mathbf{3}(\mathbf{5 0}<\mathbf{9 0} \%)$ & D/CA is mostly present & D/CA is present in submaximal ROM \\
Score $\mathbf{4}(>\mathbf{9 0} \%)$ & D/CA is always present & D/CA is present in maximal ROM \\
\hline
\end{tabular}

DIMS, Dyskinesia Impairment Mobility Scale; D/CA, dystonia/choreoathetosis; ROM, range of motion; <, lower than; $\geq$, higher than or equal; $>$, higher than; $\%$, percentage.

\subsection{Reliability and Validity}

\subsubsection{Participants}

This study included five participants aged 6-21 years old, recruited from four Flemish special education schools for children with motor disorders. Included were (1) participants with DCP diagnosed by a pediatric neurologist, (2) level IV-V for both gross motor abilities classified with the Gross Motor Function Classification System — extended and revised (GMFCS E\&R) [17] and fine manual abilities classified with the Manual Abilities Classification System-extended and revised (MACS E\&R) [17] who owned a head/foot steering wheelchair (Table A1). Excluded were individuals who (1) showed difficulties to understand and follow instructions, (2) had severe visual impairments or who (3) underwent an orthopedic or neurosurgical intervention within the last 12 months. The study was conducted in accordance with the Declaration of Helsinki, and the protocol was approved by the Medical Ethics Committee UZ KU Leuven. Informed assent and/or consent forms were signed by all participants and/or their parents.

\subsubsection{Data Collection}

Data to assess the reliability and validity of the DIMS was collected in the special education schools of the five participants, in a standardized set-up using their own head/foot steering wheelchair. Participants performed five powered mobility tasks, which were video-recorded for future scoring of the DIMS. The videos were recorded using a commercially available Sony Handycam HDR-CX405 (Sony Corporation, Tokyo, Japan) placed on a tripod on both sides of the powered mobility tasks set-up. The video recording was started manually by the researchers at the beginning of the data collection and was stopped at the end of it. The sample rate of the videos was 29 frames per second. This video-recording procedure was repeated for the powered mobility performance of each participant. 
The included tasks [18] were independent driving (i.e., T1), driving through a created corridor (i.e., T2), a 360 degree turn to the left (i.e., T3), a 360 degree turn to the right (i.e., T4) and a slalom (i.e., T5) (Figure A2). The set-up of T1, T2 and T5 was ten meters in length. The width of the corridor (T2) and the distance between cones (T5) were calculated separately per participant by measuring the length of their wheelchair and adding $50 \%$ of this length to ensure fairness in set-up, as well as comfort and safety. To meet the aims of this study, each participant was recorded twice, (i.e., Week 1 (W1) and Week 2 (W2) with two weeks' break in-between). To assess the concurrent validity of the DIMS, all five participants were additionally recorded following the standardized protocol of the existing DIS.

\subsubsection{Procedure of Scoring and Analyzing data}

The videos were independently assessed by three raters who underwent a DIS training session by the developer of the DIS (EM). Training consisted of definitions and video explanations on how to discriminate between dystonia and choreoathetosis in DCP. After the training session, the three raters independently scored ten training videos of the existing DIS to assess if they are reliable to score the DIMS videos (see Table A2).

The videos of each powered mobility task (i.e., T1, T2, T3, T4 and T5) during the two measuring weeks (W1 and W2) were scored by the three raters independently. Kinovea motion analysis software, version 0.8.15 (Kinovea, Bordeaux, France) [19] was used to cut and view the videos. Both the dystonia and choreoathetosis subscale are evaluated for duration and amplitude in five body regions. All body regions are scored during the five powered mobility tasks. Summation of the region scores gives a total score for the DIMS-D subscale and DIMS-CA subscale, each with a range from 0 to 200. The total DIMS score is the sum of the DIMS-D and DIMS-CA subscale (range 0 to 400; see Figure 1). In the current study, missing values were only $4.2 \%$ of all collected data. To account for these missing values, the summed region and subscale scores were each separately converted into percentage scores relative to their maximum score. The maximum score was accordingly adjusted (i.e., decreased) for the region or subscale scores which had missing values.

To assess the interrater reliability of the total scores of the DIMS, the DIMS-D and the DIMS-CA subscale, the scores of three raters were compared. First, all scores of W1 and W2 were converted into percentage scores. Then, the percentage scores of W1 and W2 for each rater were averaged and the obtained final score was used for the interrater reliability analysis.

To determine test-retest reliability of the DIMS, the total score of the DIMS, DIMS-D, DIMS-CA and region scores were compared between $\mathrm{W} 1$ and $\mathrm{W} 2$. The final percentage scores used for this comparison were obtained by averaging the scores of all three raters.

The internal consistency analysis was performed to assess the consistency of measuring the presence and severity of dystonia and choreoathetosis over time during powered mobility.

For the concurrent validity, neck scores of the DIMS were compared with the neck scores of the DIS for two requested voluntary activities and one rest posture. In the DIS, the first neck requested voluntary activity is neck lateroflexion and the second neck requested voluntary activity is neck rotation. The arms scores (i.e., left proximal, left distal, right proximal and right distal) of the DIMS were compared with overflow movements (scoring the contralateral side of the arm which does the required activity [9]) and rest postures scores of the DIS. The DIS and the W1 data collection of the DIMS were administered during the same week, thereby only data of W1 was used for this analysis.

\subsubsection{Statistical Analysis}

In accordance with Rigby's statistical recommendations, the intraclass correlation coefficients (ICCs) and 95\% confidence intervals (CIs) were used for the total scores and item scores of the DIMS to evaluate the interrater- and test-retest reliability [20]. To interpret the ICCs, recommendations by Monbaliu et al. were considered, with ICCs higher than 0.90 as excellent, between 0.75 and 0.90 as good, between 0.60 and 0.74 as moderately high and between 0.40 and 0.59 as moderate. ICCs less than 0.40 were indicative of low reliability [15]. Spearman's rank correlation coefficients $\left(r_{s}\right)$ were used 
to determine concurrent validity where $0.00-0.19$ was considered very weak/no correlation, $0.20-0.39$ weak, $0.40-0.59$ moderate, $0.60-0.79$ strong and 0.80 to 1.00 very strong [21]. Internal consistency was calculated by Cronbach's alpha with $\alpha=0.00$ meaning no internal consistency and $\alpha=1.00$ perfect internal consistency [21]. All statistics were calculated using IBM SPSS Statistics 25 (SPSS Inc., Chicago, IL, USA).

\section{Results}

\subsection{Interrater Reliability of the DIMS}

The ICCs and 95\% CIs of the total scale, subscale and region scores are shown in Table 2.

Moderately high to good interrater reliability was obtained for the total score of the DIMS with ICC 0.87 (95\% CI 0.35-0.99; $p=0.011)$, for the total DIMS-D with ICC 0.68 (95\% CI 0.00-0.98; $p=0.098)$ and for the total DIMS-CA with ICC 0.79 (95\% CI 0.07-0.98; $p=0.000)$. Similar interrater reliability was found for the total score of the DIMS duration factor and amplitude factor with ICC 0.62 and 0.87 respectively.

For the DIMS-D subscale, interrater reliability of the duration factor, amplitude factor and the summation of both factors were low to good, with ICCs $0.22,0.85$ and 0.68 respectively. For the DIMS-D subscale of the neck region, good interrater reliability was found for the total score with ICC 0.83 while for the DIMS-D subscale of the arm regions, ICCs ranged between 0.04 and 0.73 .

For the DIMS-CA subscale, interrater reliability of the duration factor, amplitude factor and summation of both factors were good with ICCs $0.83,0.73$ and 0.79 respectively. The interrater reliability of the total DIMS-CA for the neck was excellent with ICC 0.96 . Good to excellent interrater reliability was found for the total DIMS-CA subscale of the arm regions with ICCs ranging between 0.79 and 0.96 .

Table 2. Interrater reliability: Intraclass correlation coefficients (ICC) with $95 \%$ confidence intervals

(CI) between three raters for the Dyskinesia Impairment Mobility Scale (DIMS).

\begin{tabular}{|c|c|c|c|c|c|c|c|}
\hline & & \multicolumn{2}{|c|}{ Duration } & \multicolumn{2}{|c|}{ Amplitude } & \multicolumn{2}{|c|}{$\sum(\mathrm{D}+\mathrm{A})$} \\
\hline & & ICC & $95 \%$ CI; $p$-value & ICC & $95 \%$ CI; $p$-value & ICC & $95 \%$ CI; $p$-value \\
\hline \multicolumn{8}{|c|}{ DIMS } \\
\hline & tal score & 0.62 & $0.00-0.96 ; 0.131$ & 0.87 & $0.41-0.99 ; 0.001$ & 0.87 & $0.35-0.99 ; 0.011$ \\
\hline \multicolumn{8}{|c|}{ DIMS-D SUBSCALE } \\
\hline 1 & Neck & 0.60 & $0.00-0.96 ; 0.131$ & 0.89 & $0.40-0.99 ; 0.000$ & 0.83 & $0.27-0.98 ; 0.015$ \\
\hline 2 & Arm RP & 0.04 & $0.00-0.90 ; 0.443$ & 0.88 & $0.36-0.99 ; 0.009$ & 0.67 & $0.00-0.96 ; 0.099$ \\
\hline 3 & Arm LP & 0.15 & $0.00-0.91 ; 0.391$ & 0.87 & $0.37-0.99 ; 0.010$ & 0.67 & $0.00-0.96 ; 0.093$ \\
\hline 4 & Arm RD & 0.73 & $0.00-0.97 ; 0.074$ & 0.78 & $0.13-0.97 ; 0.006$ & 0.86 & $0.39-0.98 ; 0.005$ \\
\hline 5 & Arm LD & 0.57 & $0.00-0.96 ; 0.171$ & 0.97 & $0.87-0.99 ; 0.000$ & 0.89 & $0.37-0.99 ; 0.009$ \\
\hline & tal score & 0.22 & $0.00-0.93 ; 0.370$ & 0.85 & $0.35-0.98 ; 0.008$ & 0.68 & $0.00-0.98 ; 0.098$ \\
\hline \multicolumn{8}{|c|}{ DIMS-CA SUBSCALE } \\
\hline 1 & Neck & 0.96 & $0.55-0.99 ; 0.000$ & 0.97 & $0.75-0.99 ; 0.000$ & 0.96 & $0.63-0.99 ; 0.000$ \\
\hline 2 & Arm RP & 0.96 & $0.79-0.99 ; 0.000$ & 0.80 & $0.20-0.98 ; 0.011$ & 0.79 & $0.09-0.98 ; 0.001$ \\
\hline 3 & Arm LP & 0.98 & $0.88-0.99 ; 0.000$ & 0.90 & $0.25-0.99 ; 0.000$ & 0.96 & $0.65-0.99 ; 0.000$ \\
\hline 4 & Arm RD & 0.92 & $0.33-0.99 ; 0.000$ & 0.81 & $0.16-0.98 ; 0.000$ & 0.87 & $0.24-0.99 ; 0.000$ \\
\hline 5 & Arm LD & 0.92 & $0.45-0.99 ; 0.000$ & 0.83 & $0.13-0.98 ; 0.000$ & 0.88 & $0.23-0.99 ; 0.000$ \\
\hline \multicolumn{2}{|c|}{ Total score } & 0.83 & $0.13-0.98 ; 0.000$ & 0.73 & $0.04-0.97 ; 0.000$ & 0.79 & $0.07-0.98 ; 0.000$ \\
\hline
\end{tabular}

DIMS, Dyskinesia Impairment Mobility Scale; $\sum(\mathrm{D}+\mathrm{A})$, summation of duration and amplitude factors; ICC, intraclass correlation coefficient; CI, confidence interval; RP, right proximal; LP, left proximal; RD, right distal; LD, left distal; \%, percentage.

\subsection{Test-Retest Reliability of the DIMS}

The ICCs and 95\% CIs of the total scale, subscale and region scores are shown in Table 3.

Moderate to excellent test-retest reliability was found for the total score of the DIMS, DIMS-D and DIMS-CA with ICCs 0.80 (95\% CI 0.00-0.98; $p=0.079), 0.93(95 \%$ CI $0.42-0.99 ; p=0.000)$ and 0.51 (95\% CI 0.00-0.95; $p=0.283$ ). Good test-retest reliability was found for the total DIMS duration factor and amplitude factor with ICCs 0.76 and 0.84 respectively. 
For the DIMS-D subscale, excellent test-retest reliability was found for the duration factor, amplitude factor and summation of both, having ICCs of $0.90,0.94$, and 0.93 respectively. For the DIMS-D subscale of the neck region, excellent test-retest reliability was found for the total score with ICC 0.92 while for the DIMS-D subscale of the arm regions, ICCs ranged between 0.69 and 0.96 .

For the DIMS-CA subscale, the ICCs for the total duration factor, amplitude factor and summation of both were $0.38,0.61$ and 0.51 respectively. The ICC of the total DIMS-CA subscale for the neck region was 0.96 whereas for the arm regions, ICCs ranged between $0.00^{*}$ and 0.83 . SPSS reported negative ICC scores, most likely related to the relatively small between-subject variation compared to within-subject variation, however as negative ICCs are not theoretically possible [22], (the ICC score was changed to $0.00^{*}$ [ [23]. For this reason, an additional analysis was performed excluding the DIMS-CA arm LP scores. DIMS-CA subscale ICC scores varied from moderately high to good when excluding left proximal arm scores (see Appendix C, Table A3).

Table 3. Test-retest reliability: Intraclass Correlation Coefficients (ICC) with 95\% Confidence Interval (CI) between two measuring weeks for the Dyskinesia Impairment Mobility Scale (DIMS).

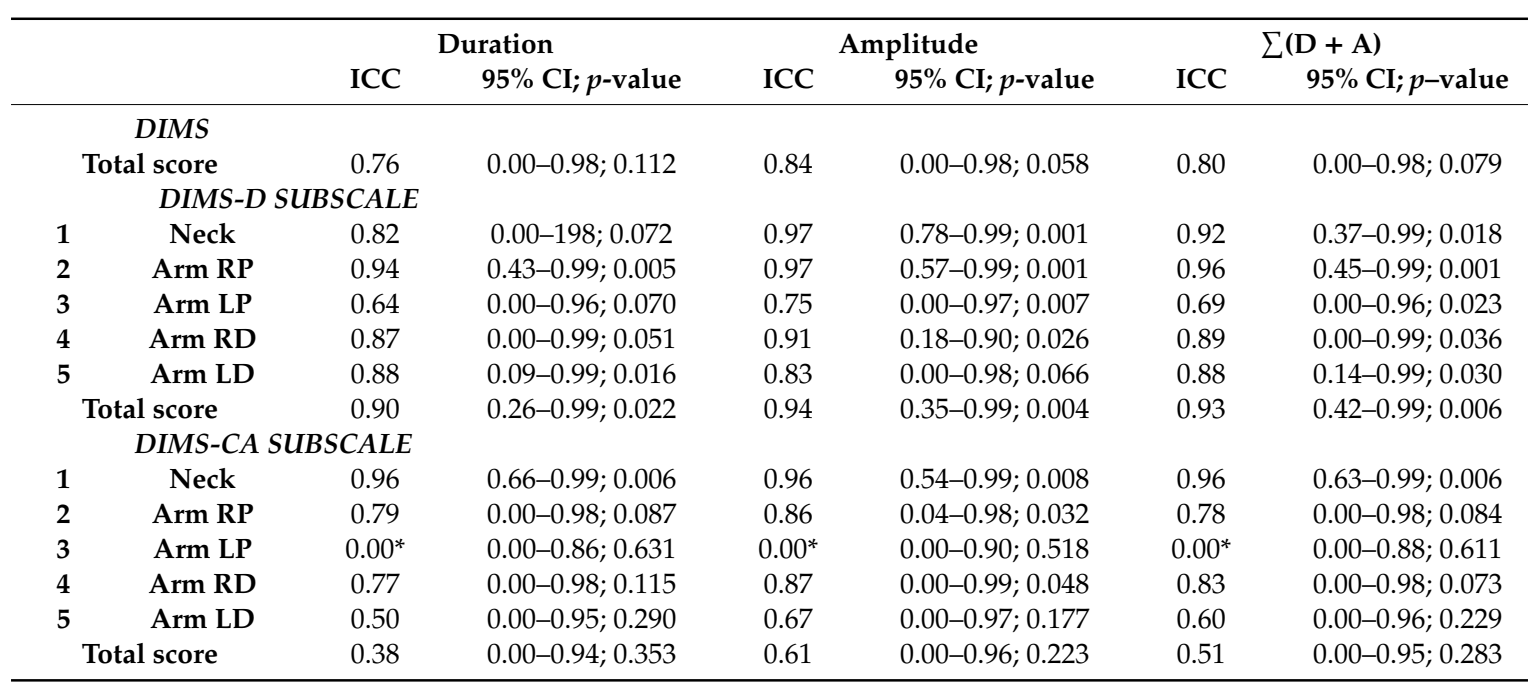

DIMS, Dyskinesia Impairment Mobility Scale; $\sum(\mathrm{D}+\mathrm{A})$, summation of duration and amplitude factors; ICC, intraclass correlation coefficient; $\mathrm{CI}$, confidence interval; RP, right proximal; LP, left proximal; RD, right distal; LD, left distal; * , negative ICC; \%, percentage.

\subsection{Internal Consistency}

Cronbach's alphas for the total DIMS, DIMS-D subscale and DIMS-CA subscale were $\alpha=0.81$, $\alpha=0.80$ and $\alpha=0.69$ respectively. For the total DIMS-D subscale, $\alpha=0.80$ was obtained for both duration factor and amplitude factor. For the total DIMS-CA subscale, $\alpha=0.71$ was found for the duration factor and $\alpha=0.66$ for the amplitude factor.

\subsection{Concurrent Validity}

For the concurrent validity of the neck region, DIMS-D subscale showed moderate correlation $\left(r_{s}\right.$ $=0.4195 \%$ CI, $0.00-0.95, p=0.003)$ while DIMS-CA subscale showed weak correlation $\left(r_{s}=0.3395 \%\right.$ CI $0.00-0.94, p=0.018$ ) with the DIS scores of the second requested voluntary activity (i.e., neck rotation). No correlation was found with the first DIS requested voluntary activity (i.e., neck lateroflexion) or with DIS neck rest postures.

For the arm RP region, DIMS-D subscale showed strong correlations $\left(r_{s}=0.6395 \%\right.$ CI $0.00-0.97$, $p=0.000)$ and DIMS-CA showed moderate correlations $\left(r_{s}=0.46(95 \%\right.$ CI $0.00-0.95, p=0.001)$ with arm RP rest postures of the DIS. No correlations were found with the DIS overflow movements.

For the arm RD region, DIMS-D subscale showed a strong correlation $r_{s}=0.6395 \%$ CI $0.00-0.97$, $p=0.000)$ with DIS rest postures and no correlation with DIS overflow movements. The DIMS-CA 
subscale showed weak correlation $\left(r_{s}=0.2995 \%\right.$ CI $\left.0.00-0.93, p=0.049\right)$ with DIS overflow movements and no correlation with DIS rest postures.

For the arm LP region, both the DIMS-D and the DIMS-CA subscale showed moderate correlations of $r_{s}=0.45(95 \%$ CI $0.00-0.95, p=0.001)$ and $r_{s}=0.41$ (95\% CI $\left.0.00-0.95, p=0.003\right)$ only with DIS rest postures.

For the arm LD region, both the DIMS-D $\left(r_{s}=0.2995 \%\right.$ CI $\left.0.00-0.93, p=0.057\right)$ and the DIMS-CA $\left(r_{s}\right.$ $=0.3795 \% \mathrm{CI} 0.00-0.94, p=0.011$ ) subscale showed weak correlations only with the DIS rest postures.

\section{Discussion}

This study aimed to develop the DIMS, a new tool to measure presence and severity of both dystonia and choreoathetosis during powered mobility tasks in DCP. The DIMS is an adapted standardized protocol of the DIS which is a reliable and valid tool to measure presence and severity of dystonia and choreoathetosis during requested but voluntary activities and rest postures. The outcomes of this study indicate that DIMS is a reliable and valid measurement tool to determine presence and severity of dystonia and choreoathetosis during powered mobility. A moderately high to good interrater reliability was found for the total score of the DIMS, the DIMS-D and the DIMS-CA with correlation coefficients of $0.87,0.68$ and 0.79 respectively. Good interrater reliability was found for the total DIMS duration factor and amplitude factor with coefficients of 0.83 and 0.73 . In addition, internal consistency scores were moderately high to good. Finally, concurrent validity showed during mobility tasks significant correlations with rest postures in the arm region, and with voluntarily, requested activity in the neck region.

Interrater reliability scores are good, in particular for DIMS total and DIMS-CA. The reliability of DIMS total is in line with both the interrater reliability of junior and senior physiotherapists [15], implying that the DIMS can be reliably used in the future by all clinicians, regardless of their work experience. Interrater reliability scores of DIMS-D duration are relatively lower as is in line with previous literature $[14,15]$. Visual inspection of the raw data showed a much lower variability in the scores of the duration factor than the scores of the amplitude factor. In addition, low DIMS-D duration scores primarily occur in proximal arm regions, while scores in neck and distal arm regions are still moderate to moderately high. This might be because the visibility of the proximal arms is slightly lower due to the sitting position of the participants while driving the wheelchair in comparison to the distal arm region and neck region. Similarly, this may explain why scoring the duration of dystonia for the proximal arms is more difficult than scoring its amplitude, which is clinically easier to see and evaluate [14]. Interestingly, for the proximal arm regions, an excellent interrater reliability was found for the duration factor of the DIMS-CA. This is likely due to the hyperkinetic nature of choreoathetosis, which is easier to observe than the hypertonic nature of dystonia [8]. The summation of both factors revealed a good interrater reliability for the DIS-D region scores, implying a reliable measure of dystonia for both voluntary and overflow movements during powered mobility. In this respect, higher scores for the duration factor as opposed to the amplitude factor were obtained for the DIMS-CA subscale, which is in line with previous research $[14,15]$. The good to excellent interrater reliability of the DIMS-CA duration and amplitude factors shows that presence and severity of choreoathetosis can be reliably measured during powered mobility.

The current study of the DIMS is the first to assess test-retest reliability in measuring presence and severity of movement disorders in DCP. The test-retest reliability of the total DIMS score, DIMS-D subscale and DIMS-CA subscale was moderate to excellent, with correlation coefficients of $0.80,0.93$ and 0.51 respectively. The test-retest reliability for the total duration factor and amplitude factor of the DIMS was also good, with coefficients 0.76 and 0.84 . Excellent test-retest reliability was obtained for the DIMS-D subscale both for the duration factor, amplitude factor and the summation of both factors. In-depth analysis of the DIMS-D region scores showed moderately high to excellent test-retest reliability for all constructs, which implies that the presence and severity of dystonia during powered mobility can be reliably measured over time, both for the voluntary and overflow movements. 
Test-retest reliability was lower for the DIMS-CA subscale when compared to the DIMS-D subscale. These low test-retest coefficients of the DIMS-CA may be because of scores at the proximal part of the left arm (i.e., arm LP). This is most likely related to the relatively small between-subject variation compared to within-subject variation of the different independent raters [23]. To explore in more depth, the arm LP scores for the DIMS-CA subscale, the raw data of the arm LP was visually inspected. This informed that for two of the participants, the presence and severity of choreoathetosis indeed changed drastically when comparing one week to the other. For one participant, the presence and severity of choreoathetosis largely decreased, while for the other participant, a large increase was seen. This could be an explanation of the obtained ICCs for the arm LP. An additional test-retest statistical analysis for the DIMS-CA subscale was performed by excluding the scores of the arm LP (Table A2). Consequently, higher test-retest reliability coefficients were obtained for the total DIMS-CA subscale, total duration factor and amplitude factor, including higher test-retest coefficients of the total DIMS. The test-retest coefficients of the DIMS-CA for the remaining neck and arm regions (i.e., arm RP, arm RD and arm LD) were moderate to excellent, suggesting that the DIMS can reliably measure the presence and severity of choreoathetosis over time.

The internal consistency of the DIMS was good, with Cronbach's $\alpha$ ranging between 0.69 and 0.81 for the total score of the DIMS and the DIMS-D and DIMS-CA subscales. This indicates a stable rating construct in measuring the movement disorders during mobility tasks in children with DCP which is comparable with the internal consistency of the DIS scale $[14,15]$. Although the DIMS could potentially become a tool to use in longitudinal follow-ups or intervention studies, future research should also focus in assessing its responsiveness.

The concurrent validity was assessed between the DIMS scores and the DIS scores (i.e., requested voluntary activities, rest postures and overflow movements) of the same participants. Interestingly, for the neck region, both dystonia and choreoathetosis scores of the DIMS were correlated with the DIS neck rotation requested voluntary activity and no correlation was obtained with the DIS neck lateroflexion or DIS neck rest postures. The participants indeed use a rotation of the neck to be able to steer the wheelchair to the right or to the left; therefore, the obtained findings correspond with observations and knowledge from clinical practice. On the contrary, the DIMS scores of the arms region showed correlations with the arms rest postures of the DIS and not with overflow movements, except for the right distal arm. Research suggests that presence and severity of dystonia and choreoathetosis increase during requested voluntary activities as opposed to rest [24]. As the arms are not doing any goal-directed activity while operating with the head/foot steering wheelchair, it is highly likely that the presence and severity of the movement disorders in the arms during powered mobility corresponds more to the resting postures of the participants rather than requested voluntary activities which are challenging to perform.

This study is the first to present a tool that reliably measures presence and severity of dystonia and choreoathetosis during powered mobility in children and youth with DCP. The DIMS and generated insights from its use have the potential to inform and help clinicians set up more a more straightforward and efficient mobility training based on structured guidelines. As a first tool to measure movement disorders relevant for DCP during powered mobility tasks, future studies using the DIMS will yield important insights on powered mobility in DCP, which is of crucial importance and yet underexplored in the target population. Nevertheless, this study warrants some reflections to consider. First, the sample size of this study is small, considering the challenging inclusion criteria like the rare DCP diagnosis and the use of their own head/foot steering wheelchair. However, the number of tasks and regions scored by three independent raters gives confidence in the reported results. The low number of included participants has likely a relatively negative impact on the reliability scores in comparison to a higher number of participants. That is, it is plausible that with more scores included in the reliability statistical analysis, the obtained reliability coefficients would be higher. Still, given the reported outcomes in this study, the DIMS can be perceived a reliable and valid measurement tool to measure presence and severity of dystonia and choreoathetosis during powered mobility. Second, the age range 
of the participants and their years of experience in driving a head/foot steering system is large. Again, this could have negatively affected our outcomes in terms of the reliability scores and concurrent validity scores of the DIMS. Therefore, future studies are also advised to consider categorizing the sample based on their age and years of experience to explore any differences and generate more in-depth insights. Finally, due to the importance of the quality of the video recordings, we would like to strongly advise to use high-quality cameras with a high resolution and zoom-in function.

\section{Conclusions}

This study developed the Dyskinesia Impairment Mobility Scale (DIMS), an adapted protocol of the existing Dyskinesia Impairment Scale (DIS), to evaluate the presence and severity of dystonia and choreoathetosis during powered mobility tasks in individuals with DCP. The DIMS is a reliable and valid measurement tool to determine presence and severity of dystonia and choreoathetosis during powered mobility. The DIMS showed moderately high to good interrater reliability, good internal consistency and moderate to excellent test-retest reliability for the voluntary and overflow movements. Concurrent validity showed, during mobility tasks, significant correlations with rest postures in the arm region, and with requested voluntary activity in the neck region.

In clinical practice, the DIMS could be a promising tool to assess and evaluate the presence and severity of dystonia and choreoathetosis during powered mobility tasks, and assist in accelerating the learning process of using a powered mobility wheelchair by providing baseline profiles and a reliable longitudinal follow-up of the severity of the movement disorders which greatly impact mobility. Increased insights in clinical movement disorders during powered mobility may generate knowledge on the powered mobility driving patterns which can be used by the clinicians to tailor individualized mobility training programs. Furthermore, in future research, the DIMS can be used to explore clinical patterns of dystonia and choreoathetosis during steering and be used as an evaluation tool of future mobility intervention studies. Moreover, the DIMS could inform on the impact of factors such as fatigue, stress or emotional arousal on movement disorders during powered mobility, leading to increased insights that could assist in the development of more straightforward mobility training guidelines.

Author Contributions: Conceptualization, S.B., I.G.N., S.G., J.-M.A., H.H. and E.M; methodology, S.B., I.G.N., M.K., J.-M.A. and E.M.; validation, S.B.; formal analysis, S.B.; investigation, S.B., I.G.N., S.G. and I.V.; resources, S.B. and E.M.; data curation, S.B. and M.K.; writing-original draft preparation, S.B.; writing-review and editing, M.K., I.V., I.G.N., S.G., J.-M.A., H.H. and E.M.; visualization, S.B.; supervision, E.M., M.K., J.-M.A and H.H.; project administration, S.B. and E.M.; funding acquisition, E.M., J.-M.A. and H.H.

Funding: This research was funded by a C3 grant from the Research Council of KU Leuven, grant number C32/17/056.

Acknowledgments: The authors express immense gratitude to the participants who were included in this study and their supportive parents. A special thank you goes to the therapists of the participants for their invaluable help from the screening process to data collection: Bart Moerman, physical therapist, Dominiek Savio Institute, Gits, Belgium; Ms. Heleen Soens, physical therapist, Dominiek Savio Institute, Gits, Belgium; and Veerle Janssens, occupational therapist in Sint Jozef Institute (Heder), Antwerp. Acknowledged are the two master thesis students: Alice Di Berardino and Hannah Rooseleers for their help and hard work in scoring the participants as part of their master thesis project. The authors thank professor Jan Deklerck for the valuable feedback on the methodology and statistical analysis of this study.

Conflicts of Interest: The authors declare no conflict of interest. The funders had no role in the design of the study; in the collection, analyses, or interpretation of data; in the writing of the manuscript, or in the decision to publish the results. 


\section{Appendix A}

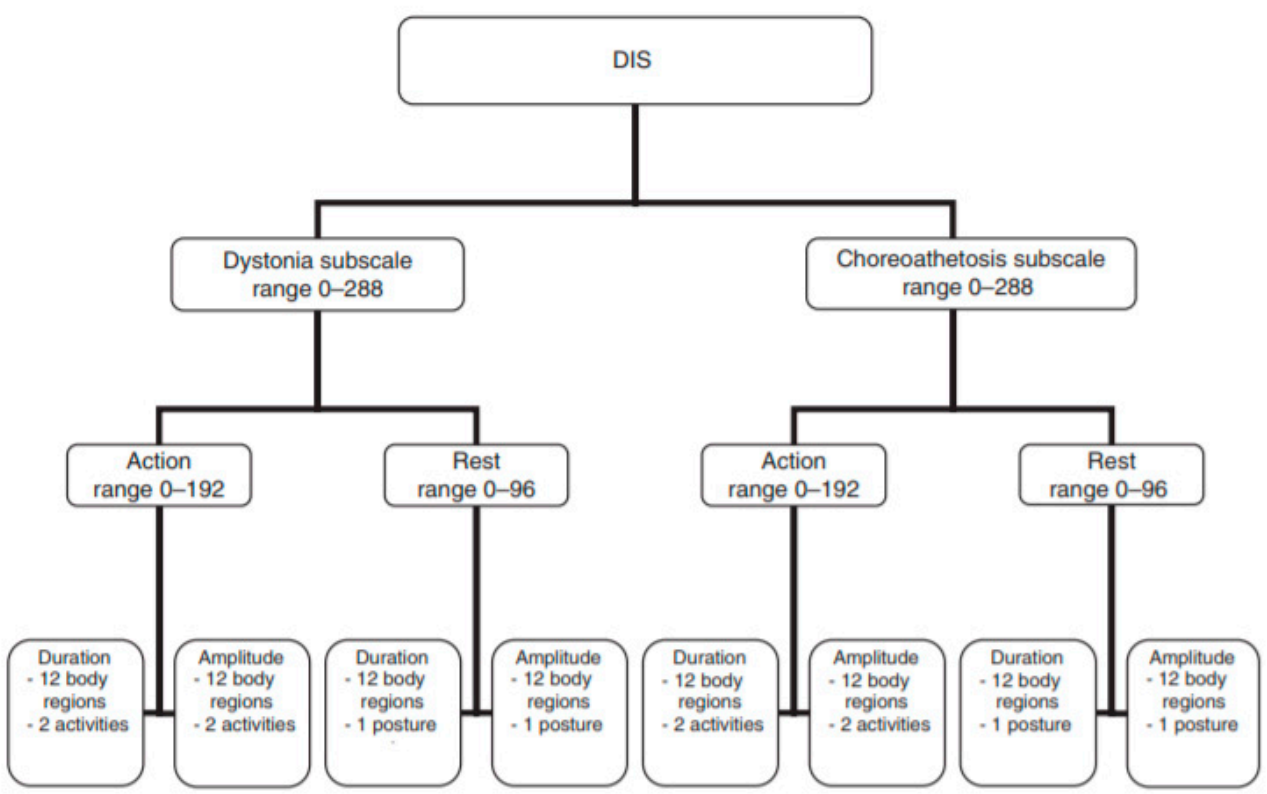

Figure A1. Diagram of the Dyskinesia Impairment Scale (DIS) protocol.

\section{Appendix B}

Table A1. Participants' characteristics.

\begin{tabular}{cccccc}
\hline & $\begin{array}{c}\text { Gender } \\
\text { (M/F) }\end{array}$ & Age (yo) & GMFCS & MACS & $\begin{array}{c}\text { Experience with Powered } \\
\text { Mobility (Years) }\end{array}$ \\
\hline P01 & M & 19 & 4 & 4 & 10 \\
P02 & M & 16 & 4 & 4 & 6 \\
P03 & F & 6 & 4 & 4 & 1.5 \\
P04 & F & 21 & 5 & 4 & 6 \\
P05 & F & 8 & 4 & 5 & 1 \\
\hline
\end{tabular}

P, participant; M, male; F, female; yo, year old; GMFCS, Gross Motor Function Classification System; MACS, Manual Ability Classification Scale.

\section{Appendix C}

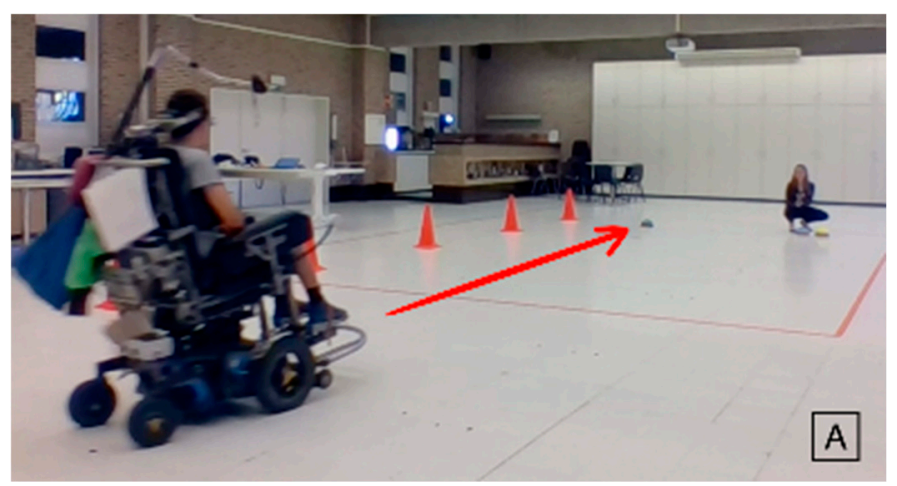

Figure A2. Cont. 

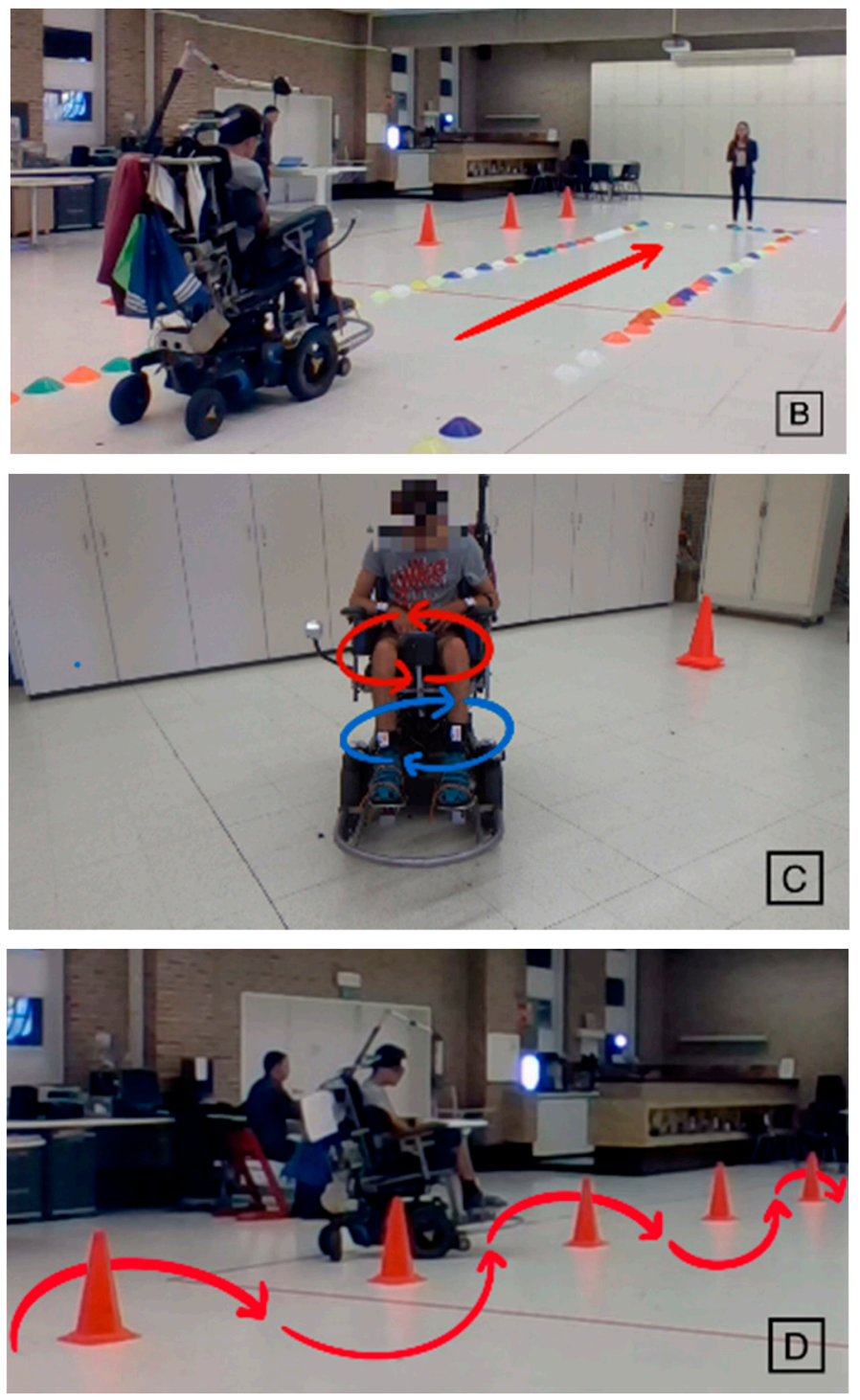

Figure A2. Data collection set-up with five standardized powered mobility tasks: (A) T1, Independent driving; (B) T2, driving through a created corridor; (C) T3, $360^{\circ}$ turn to the left (red arrow); T4, 360 turn to the right (blue arrow); (D) T5, slalom.

\section{Appendix D}

Table A2. Interrater reliability of DIS Training: Intraclass Correlation Coefficients (ICC) with $95 \%$ confidence interval $(\mathrm{CI})$ between three raters for the total scores.

\begin{tabular}{ccccccc}
\hline & \multicolumn{2}{c}{ Duration } & \multicolumn{2}{c}{ Amplitude } & \multicolumn{2}{c}{$\sum$ (D + A) } \\
\hline & ICC & $\mathbf{9 5 \% ~ C I ; ~} \boldsymbol{p}$-value & ICC & $\mathbf{9 5 \% ~ C I ; ~} \boldsymbol{p}$-value & ICC & $\mathbf{9 5 \% ~ C I ; ~} \boldsymbol{p}$-value \\
\hline Dystonia & 0.91 & $0.74-0.98 ; 0.000$ & 0.89 & $0.69-0.97 ; 0.000$ & 0.89 & $0.70-0.97 ; 0.000$ \\
Choreoathetosis & 0.95 & $0.85-0.99 ; 0.000$ & 0.96 & $0.90-0.99 ; 0.000$ & 0.96 & $0.88-0.99 ; 0.000$ \\
DIS Training & 0.94 & $0.83-0.98 ; 0.000$ & 0.95 & $0.86-0.99 ; 0.000$ & 0.96 & $0.88-0.99 ; 0.000$ \\
\hline
\end{tabular}

ICC, Intraclass Correlation Coefficient; CI, Confidence Interval; DIS, Dyskinesia Impairment Scale; $\sum(\mathrm{D}+\mathrm{A})$, summation of duration and amplitude factors; $\%$, percentage. 


\section{Appendix E}

Table A3. Test-retest reliability: Intraclass correlation coefficients (ICC) with 95\% confidence interval (CI) between two measuring weeks for the Dyskinesia Impairment Mobility Scale, with excluded left arm proximal scores for the DIMS-CA subscale.

\begin{tabular}{|c|c|c|c|c|c|c|c|}
\hline & & \multicolumn{2}{|c|}{ Duration } & \multicolumn{2}{|c|}{ Amplitude } & \multicolumn{2}{|c|}{$\sum(\mathrm{D}+\mathrm{A})$} \\
\hline & & ICC & $95 \%$ CI; $p$-value & ICC & $95 \%$ CI; $p$-value & ICC & $95 \%$ CI; $p$-value \\
\hline \multicolumn{8}{|c|}{ DIMS } \\
\hline \multicolumn{8}{|c|}{ DIMS-D SUBSCALE } \\
\hline 1 & Neck & 0.82 & $0.00-198 ; 0.072$ & 0.97 & $0.78-0.99 ; 0.001$ & 0.92 & $0.37-0.99 ; 0.018$ \\
\hline 2 & Arm RP & 0.94 & $0.43-0.99 ; 0.005$ & 0.97 & $0.57-0.99 ; 0.001$ & 0.96 & $0.45-0.99 ; 0.001$ \\
\hline 4 & Arm RD & 0.87 & $0.00-0.99 ; 0.051$ & 0.91 & $0.18-0.90 ; 0.026$ & 0.89 & $0.00-0.99 ; 0.036$ \\
\hline 5 & Arm LD & 0.88 & $0.09-0.99 ; 0.016$ & 0.83 & $0.00-0.98 ; 0.066$ & 0.88 & $0.14-0.99 ; 0.030$ \\
\hline & l score & 0.90 & $0.26-0.99 ; 0.022$ & 0.94 & $0.35-0.99 ; 0.004$ & 0.93 & $0.42-0.99 ; 0.006$ \\
\hline \multicolumn{8}{|c|}{ DIMS-CA SUBSCALE } \\
\hline 1 & Neck & 0.96 & $0.66-0.99 ; 0.006$ & 0.96 & $0.54-0.99 ; 0.008$ & 0.96 & $0.63-0.99 ; 0.006$ \\
\hline \multicolumn{2}{|c|}{ Total score } & 0.62 & $0.00-0.96 ; 0.217$ & 0.75 & $0.00-0.98 ; 0.130$ & 0.71 & $0.00-0.97 ; 0.160$ \\
\hline
\end{tabular}

DIMS, Dyskinesia Impairment Mobility Scale; $\sum(\mathrm{D}+\mathrm{A})$, summation of duration and amplitude factors; ICC, Intraclass Correlation Coefficient; CI, Confidence Interval; RP, Right Proximal; L, Left Proximal; RD, Right Distal; LD, Left Distal; ${ }^{*}$, negative ICCs excluded from the analysis of DIMS-CA subscale; $\%$, percentage.

\section{References}

1. Livingstone, R.; Field, D. Systematic review of power mobility outcomes for infants, children and adolescents with mobility limitations. Clin. Rehabil. 2014, 28, 954-964. [CrossRef] [PubMed]

2. Casey, J.; Paleg, G.; Livingstone, R. Facilitating Child Participation through Power Mobility. Br. J. Occup. Ther. 2013, 76, 158-160. [CrossRef]

3. Livingstone, R.; Field, D. The child and family experience of power mobility: A qualitative synthesis. Dev. Med. Child Neurol. 2015, 57, 317-327. [CrossRef] [PubMed]

4. Livingstone, R. A critical review of powered mobility assessment and training for children. Disabil. Rehabil. Assist. Technol. 2010, 5, 392-400. [CrossRef] [PubMed]

5. Rodby-Bousquet, E.; Paleg, G.; Casey, J.; Wizert, A.; Livingstone, R. Physical risk factors influencing wheeled mobility in children with cerebral palsy: A cross-sectional study. BMC Pediatr. 2016, 16. [CrossRef]

6. Sellier, E.; Platt, M.J.; Andersen, G.L.; Krageloh-Mann, I.; De La Cruz, J.; Cans, C. Decreasing prevalence in cerebral palsy: A multi-site European population-based study, 1980 to 2003. Dev. Med. Child Neurol. 2016, 58, 85-92. [CrossRef] [PubMed]

7. Rosenbaum, P.; Paneth, N.; Leviton, A.; Goldstein, M.; Bax, M.; Damiano, D.; Dan, B.; Jacobsson, B. A report: The definition and classification of cerebral palsy April 2006. Dev. Med. Child Neurol. Suppl. 2007, 109, 8-14. [PubMed]

8. Monbaliu, E.; Himmelmann, K.; Lin, J.P.; Ortibus, E.; Bonouvrie, L.; Feys, H.; Vermeulen, R.J.; Dan, B. Clinical presentation and management of dyskinetic cerebral palsy. Lancet Neurol. 2017, 16, 741-749. [CrossRef]

9. Vanmechelen, I.; Bekteshi, S.; Bossier, K.; Feys, H.; Deklerck, J.; Monbaliu, E. Presence and severity of dystonia and choreoathetosis overflow movements in participants with dyskinetic cerebral palsy and their relation with functional classification scales. Disabil.Rehabil 2019, 1-8. [CrossRef] [PubMed]

10. Monbaliu, E.; De La Pena, M.G.; Ortibus, E.; Molenaers, G.; Deklerck, J.; Feys, H. Functional outcomes in children and young people with dyskinetic cerebral palsy. Dev. Med. Child Neurol. 2017, 59, 634-640. [CrossRef] [PubMed]

11. Rodby-Bousquet, E.; Hägglund, G. Use of manual and powered wheelchair in children with cerebral palsy: A cross-sectional study. BMC Pediatr. 2010, 10, 59. [CrossRef] [PubMed] 
12. Livingstone, R.; Paleg, G. Practice considerations for the introduction and use of power mobility for children. Dev. Med. Child Neurol 2014, 56, 210-221. [CrossRef] [PubMed]

13. Giesbrecht, E.M.; Wilson, N.; Schneider, A.; Bains, D.; Hall, J.; Miller, W.C. Preliminary Evidence to Support a "Boot Camp" Approach to Wheelchair Skills Training for Clinicians. Arch. Phys. Med. Rehabil. 2015, 96, 1158-1161. [CrossRef] [PubMed]

14. Monbaliu, E.; Ortibus, E.; De Cat, J.; Dan, B.; Heyrman, L.; Prinzie, P.; De Cock, P.; Feys, H. The Dyskinesia Impairment Scale: A new instrument to measure dystonia and choreoathetosis in dyskinetic cerebral palsy. Dev. Med. Child Neurol 2012, 54, 278-283. [CrossRef] [PubMed]

15. Monbaliu, E.; Ortibus, E.; Prinzie, P.; Dan, B.; De Cat, J.; De Cock, P.; Feys, H. Can the Dyskinesia Impairment Scale be used by inexperienced raters? A reliability study. Eur. J. Paediatr. Neurol. 2013, 17, $238-247$. [CrossRef] [PubMed]

16. Vanmechelen, I.; Dan, B.; Feys, H.; Monbaliu, E. Test-retest reliability of the Dyskinesia Impairment Scale: Can dystonia and choreoathetosis be reliably measured over time in dyskinetic cerebral palsy? Dev. Med. Child Neurol. 2019, under review.

17. Palisano, R.J.; Avery, L.; Gorter, J.W.; Galuppi, B.; McCoy, S.W. Stability of the Gross Motor Function Classification System, Manual Ability Classification System, and Communication Function Classification System. Dev. Med. Child Neurol. 2018, 60, 1026-1032. [CrossRef] [PubMed]

18. Kirby, R.L.; Smith, C.; Routhier, F.; Best, K.L.; Cowan, R.; Giesbrecht, E.; Koontz, A.; MacKenzie, D.; Mortenson, B.; Parker, K.; et al. The Wheelchair Skills Program Manual Version 5.0. Available online: www.wheelchairskillsprogram.ca/eng/manual.php (accessed on 15 February 2018).

19. Kinovea 0.8.15. Available online: www.kinovea.org (accessed on 10 September 2018).

20. RIGBY, A.S. Statistical recommendations for papers submitted to Developmental Medicine \& Child Neurology. Dev. Med. Child Neurol. 2010, 52, 299-304. [CrossRef] [PubMed]

21. Portney, L.G.; Watkins, M.P. Foundations of Clinical Research: Applications to Practice, 3rd ed.; Pearson/Prentice Hall: Upper Saddle River, NJ, USA, 2014.

22. Gireaudeau, B. Negative Values of the Intraclass Correlation Coefficients Are Not Theoretically Possible. J. Clin. Epidemiol. 1996, 49, 1205-1206. [CrossRef]

23. London King's College. Available online: https://www.kcl.ac.uk/ioppn/depts/BiostatisticsHealthInformatics/ SAS/faqs (accessed on 28 March 2019).

24. Monbaliu, E.; de Cock, P.; Ortibus, E.; Heyrman, L.; Klingels, K.; Feys, H. Clinical patterns of dystonia and choreoathetosis in participants with dyskinetic cerebral palsy. Dev. Med. Child Neurol. 2016, 58, 138-144. [CrossRef] [PubMed]

(C) 2019 by the authors. Licensee MDPI, Basel, Switzerland. This article is an open access article distributed under the terms and conditions of the Creative Commons Attribution (CC BY) license (http://creativecommons.org/licenses/by/4.0/). 